Report to the ILC World-wide Study

\title{
Physics Benchmarks for the ILC Detectors
}

\author{
M. Battaglia \\ Dept. of Physics, University of California and LBNL, Berkeley, CA 94720 USA \\ T. Barklow, M. E. Peskin \\ SLAC, Stanford CA 94309 USA \\ Y. Okada \\ KEK, Tsukuba-shi, Ibaraki-ken 305-0801 JAPAN \\ S. Yamashita \\ International Center for Elementary Particle Physics, University of Tokyo, Tokyo 113-0033 JAPAN \\ P. Zerwas \\ DESY, Hamburg D-22603 GERMANY

\begin{abstract}
This note presents a list of physics processes for benchmarking the performance of proposed ILC detectors. This list gives broad coverage of the required physics capabilities of the ILC experiments and suggests target accuracies to be achieved. A reduced list of reactions, which capture within a very economical set the main challenges put by the ILC physics program, is suggested for the early stage of benchmarking of the detector concepts.
\end{abstract}

\section{INTRODUCTION}

Now that the RF technology of the ILC has been chosen, concepts are taking shape for the design, components, and optimization of the detectors [1, 2, 3]. At the same time, a program of R\&D on sensors and sub-detector components is being carried out worldwide. It is important to both studies to justify the design R\&D goals by the required physics capabilities of the detector. In time, it will also be important to compare the relative integral performance of the different concepts. These evaluations should be based on a standard set of reference physics processes. Even today, at the early stages of the detector design process, it is valuable to review the accuracy targets that must eventually be met for the major reactions of the linear collider physics program and to survey the full set of capabilities that a linear collider detector must have. In this note, we would like to propose a set of reference reactions that addresses these goals. We hope that this list of reactions will be a useful guidepost in the R\&D studies. We also hope that, by providing focus on specific problems, it will promote more realistic physics simulations that take detailed account of the detector response and the machine-detector interface. This will be needed for the detector designs, and it will also help in refining our assessment of the ILC physics potential.

A formal set of benchmark processes should fulfill some basic requirements. First, it should be designed so that the central physics scenarios are broadly covered. It should contain the most important reactions that give the physics justification of the ILC and illustrate how the ILC complements and extends the results expected from the LHC. Second, the benchmark processes should be robust. A benchmark should not be tailored to a singular physics problem but rather should address issues common to a variety of physics analyses. That is a benchmark should ensure that the detector criteria that it tests would be appropriate also for physics scenarios that have not been considered. Third, the effect of the performance of individual detector components on the physics results should be manifest. It is important that the benchmarks have well-defined target goals for measurement accuracy that are motivated quantitatively by the requirements of the ILC physics program and the anticipated potential of the LHC and of astrophysics experiments. 
These requirements can be addressed by examples chosen from three classes of processes: i) studies of the Higgs boson or Higgs sector; ii) studies of Supersymmetry, and iii) precision measurements in the Standard Model with indirect sensitivity to New Physics. Examples in the third class also give precise goals for measurements of beam energy and collider luminosity, measurements that will also be crucial in the precision study of new and exotic particles.

Several sets of benchmarks have been proposed over the years of the linear collider physics study. However, we feel that, as we begin a new stage in the definition of the ILC and its detectors, it is useful to comprehensively review the menu of benchmark processes. In this note we define new sets of physics reactions that we propose be used in validating the detector design and optimising its performances. This note is organised as follows. We first discuss a broad set of key physics reactions that met the goals just discussed for broad coverage of the ILC program, and that have well defined target performances. We then review the physics-detector matrix linking the performance of the various detector subsystems to the physics reactions. Finally, as a starting point for detailed studies of detector design, we propose a set of ILC measurements that offer an adequate sampling of the major challenges for the ILC detectors within as small a list of processes as possible.

\section{PHYSICS REACTIONS}

In this section, we review physics processes of interest in defining detector performance. For each quantity to be measured, we present a target accuracy. These accuracies are based on quantitatively well-defined goals of the ILC, whose limits are set either by theoretical uncertainties (as in the case of the Higgs branching fractions) or by matching the accuracy of other measurements (as in the case of the cosmology-motivated SUSY scenarios). Each goal is to be obtained by running at one of three fixed centre of mass energies $-350 \mathrm{GeV}, 500 \mathrm{GeV}$, or $1 \mathrm{TeV}$ - with the event samples expected from the ILC design $-0.5 \mathrm{ab}^{-1}, 0.5 \mathrm{ab}^{-1}, 1.0 \mathrm{ab}^{-1}$, respectively. In most cases, the reactions listed have been studied using parametric simulation and the required target accuracies have been shown to be achievable at that level.

\section{A. Higgs Boson and Higgs Sector}

The study of the Higgs sector offers a set of reactions where physics results need to be extracted with clearly defined accuracy. These reactions involve a wide variety of final states, ranging from two leptons to eight jets. The particular case of a Standard Model Higgs boson $h^{0}$ with mass $M_{h}=120 \mathrm{GeV}$ offers significant branching fractions to a large variety of different decay channels. Through this case, we can sample the main processes that will define the profile of a light Higgs boson.

\section{B. Supersymmetry}

Supersymmetry offers an enormous parameter space in which many different physics processes appear at varying levels of importance. It is often not obvious what accuracy is required for measurements of supersymmetry particle masses and properties. However, there are two circumstances in which the requirement are very clear. First, when some parameters of the superparticle spectrum can be measured at LHC, one should insist the new information from ILC signficantly improves the accuracy of this analysis. Second, when the supersymmetry parameters are such as to produce a candidate for the cosmic dark matter, one should insist that the linear collider experiments should allow one to compute the dark matter density to an accuracy that would provide a significant comparison with the determination to a few percent accuracy from measurements of the cosmic microwave background. Both arguments turn out to require high-precision mass measurements, at the level of $1 \%-0.1 \%$ accuracy, for several supersymmetry particle masses or mass differences. The various scenarios that we have included for supersymmetric dark matter emphasize different final states that range from dileptons plus missing energy to complex multi-jet 
TABLE I: Model parameters and particle masses for benchmark points taken as points in the mSUGRA model. The spectrum for these points have been computed using ISAJET $7.69[4]$ as a reference code.

\begin{tabular}{|c|l|c|c|c|c|c|c|c|c|c|c|c|c|c|c|}
\hline Point & Ref. & $\begin{array}{c}m_{0} \\
\mathrm{GeV}\end{array}$ & $\begin{array}{c}m_{1 / 2} \\
\mathrm{GeV}\end{array}$ & $\begin{array}{c}\tan \beta \\
\mathrm{GeV}\end{array}$ & $\begin{array}{c}\mu \\
\mathrm{GeV}\end{array}$ & $\begin{array}{c}M_{\text {top }} \\
\mathrm{GeV}\end{array}$ & $\begin{array}{c}M_{\tilde{\chi}_{1}^{0}} \\
\mathrm{GeV}\end{array}$ & $\begin{array}{c}M_{\tilde{\tau}_{1}} \\
\mathrm{GeV}\end{array}$ & $\begin{array}{c}M_{\tilde{\chi}_{2}^{0}} \\
\mathrm{GeV}\end{array}$ & $\begin{array}{c}M_{\tilde{\chi}_{3}^{0}} \\
\mathrm{GeV}\end{array}$ & $\begin{array}{c}M_{\tilde{e}_{R}} \\
\mathrm{GeV}\end{array}$ & $\begin{array}{c}M_{A} \\
\mathrm{GeV}\end{array}$ & $\begin{array}{c}M_{\tilde{\chi}_{1}^{+}} \\
\mathrm{GeV}\end{array}$ & $\begin{array}{c}M_{\tilde{\chi}_{2}^{+}} \\
\mathrm{GeV}\end{array}$ \\
\hline \hline 1 & SPS1a' [5, 6] & 70 & 250 & 10 & -300 & 389 & 175 & 96.1 & 109.2 & 185 & 393 & 124 & 421 & 185 & 408 \\
2 & LCC2 [6] & 3280 & 300 & 10 & 0 & 178 & 175 & 107.7 & 3251 & 166 & 190 & 3270 & 3242 & 159 & 287 \\
3 & D' [7] & 110 & 525 & 10 & 0 & 654 & 175 & 211.3 & 220.8 & 408 & 658 & 228 & 744 & 409 & 671 \\
4 & LCC4 [6] & 380 & 420 & 53 & 535 & 0 & 178 & 169.1 & 195 & 327 & 540 & 412 & 419 & 328 & 553 \\
5 & $\alpha[8]$ & 206 & 293 & 10 & 0 & 375 & 178 & 113 & 213 & 215 & 380 & 216 & 265 & 215 & 399 \\
\hline \hline 6 & $\epsilon[8]$ & 20 & 440 & 15 & -25 & 569 & 178 & 175 & 153 & 339 & 574 & 171 & 622 & 340 & 587 \\
\hline
\end{tabular}

events. In addition, we have included three special models with late-decaying particles or exceptionally small mass degeneracy. Parameters of the mSUGRA benchmark points are given in Table \ those of the alternative SUSY breaking mechanisms are given in the footnotes $\{20\}$ and $\{21\}$.

\section{Precision Tests of the Standard Model}

Some models of new physics that do not involve supersymmetry nevertheless lead to signatures similar to those of supersymmetric models. But other new physics scenarios lead to high-mass resonances that affect the standard process of $e^{+} e^{-}$annihilation indirectly. For example, models in which the Higgs bosons are heavier than the precision electroweak limit typically contain new vector resonances.

The ILC has very powerful capabilities to analyze such models through precision measurements on the affected processes. The sensitivity of these measurements to heavy resonances typically exceeds the sensitivity of the LHC to observe the resonance directly. Further, if a heavy resonance is observed at the LHC, precision measurement of the anomalies it produces in Standard Model reactions will give us detailed information about its couplings. Thus, we have included precision measurements of all of the major Standard Model processes as elements of our list of benchmark reactions. We have also included two scenarios that illustrate different manifestations of new physics, the ADD scenario of large extra dimensions leading to single-photon missing energy events, and a scenario in which a graviton resonance in the Randall-Sundrum scenario is directly accessible at $1 \mathrm{TeV}$.

\section{DETECTOR SUBSYSTEMS}

In this section, we review the various subsystems whose performance contributes to the overall detector design. We explain which benchmark processes are especially important to the optimization of each subsystem.

\section{A. Vertexing}

The branching fractions of a light Higgs boson to $b \bar{b}, c \bar{c}$ still form the core of the case for excellent vertex detector performance at the ILC. Accurate measurements of these branching fractions are needed to test the Higgs mechanism of mass generation. Recent progress in the determination of heavy quark masses using spectral distribution moments in semileptonic $b$ decays has provided a significant reduction in $M_{q}$ uncertainties, thus motivating an even better experimental accuracy.

Vertexing may play a role in $\tau$ identification in the measurement of the branching fraction for $h \rightarrow \tau^{+} \tau^{-}$or in isolating the stau signal in $e^{+} e^{-} \rightarrow \tilde{\tau}_{1} \tilde{\tau}_{1} \rightarrow \tilde{\chi}_{1}^{0} \tilde{\chi}_{1}^{0} \tau^{+} \tau^{-}$. Precise vertexing is also useful in efficiently selecting the sign of a $b$ or $c$ quark through the measurement of the net charge of the displaced vertex. The vertex charge technique 
TABLE II: Benchmark reactions for the evaluation of ILC detectors

\begin{tabular}{|c|c|c|c|c|c|c|}
\hline & $\begin{array}{l}\text { Process and } \\
\text { Final states }\end{array}$ & $\begin{array}{l}\text { Energy } \\
(\mathrm{TeV})\end{array}$ & Observables & $\begin{array}{l}\text { Target } \\
\text { Accuracy }\end{array}$ & $\begin{array}{l}\text { Detector } \\
\text { Challenge }\end{array}$ & Notes \\
\hline Higgs & $\begin{array}{l}e e \rightarrow Z^{0} h^{0} \rightarrow \ell^{+} \ell^{-} X \\
e e \rightarrow Z^{0} h^{0}, h^{0} \rightarrow b \bar{b} / c \bar{c} / \tau \tau \\
e e \rightarrow Z^{0} h^{0}, h^{0} \rightarrow W W^{*} \\
e e \rightarrow Z^{0} h^{0} / h^{0} \nu \bar{\nu}, h^{0} \rightarrow \gamma \gamma \\
e e \rightarrow Z^{0} h^{0} / h^{0} \nu \bar{\nu}, h^{0} \rightarrow \mu^{+} \mu^{-} \\
e e \rightarrow Z^{0} h^{0}, h^{0} \rightarrow \text { invisible } \\
e e \rightarrow h^{0} \nu \bar{\nu} \\
e e \rightarrow t \bar{t} h^{0} \\
e e \rightarrow Z^{0} h^{0} h^{0}, h^{0} h^{0} \nu \bar{\nu}\end{array}$ & $\begin{array}{l}0.35 \\
0.35 \\
0.35 \\
1.0 \\
1.0 \\
0.35 \\
0.5 \\
1.0 \\
0.5 / 1.0 \\
\end{array}$ & $\begin{array}{l}\mathrm{M}_{\mathrm{recoil}}, \sigma_{Z h}, \mathrm{BR}_{b b} \\
\text { Jet flavour }, \text { jet }(E, \vec{p}) \\
\mathrm{M}_{Z}, \mathrm{M}_{W}, \sigma_{q q W W^{*}} \\
M_{\gamma \gamma} \\
M_{\mu \mu} \\
\sigma_{q q E} \\
\sigma_{b b \nu \nu}, M_{b b} \\
\sigma_{t t h} \\
\sigma_{Z h h}, \sigma_{\nu \nu h h}, M_{h h}\end{array}$ & $\begin{array}{l}\delta \sigma_{Z h}=2.5 \%, \delta \mathrm{BR}_{b b}=1 \% \\
\delta \mathrm{M}_{h}=40 \mathrm{MeV}, \quad \delta\left(\sigma_{Z h} \times \mathrm{BR}\right)=1 \% / 7 \% / 5 \% \\
\delta\left(\sigma_{Z h} \times \mathrm{BR}_{W W^{*}}\right)=5 \% \\
\delta\left(\sigma_{Z h} \times \mathrm{BR}_{\gamma \gamma}\right)=5 \% \\
5 \sigma \text { Evidence for } M_{h}=120 \mathrm{GeV} \\
5 \sigma \text { Evidence for } \mathrm{BR}_{\text {invisible }}=2.5 \% \\
\delta\left(\sigma_{\nu \nu h} \times \mathrm{BR}_{b b}\right)=1 \% \\
\delta g_{t t h}=5 \% \\
\delta g_{h h h}=20 / 10 \%\end{array}$ & $\begin{array}{l}\mathrm{T} \\
\mathrm{V} \\
\mathrm{C} \\
\mathrm{C} \\
\mathrm{T} \\
\mathrm{C} \\
\mathrm{C} \\
\mathrm{C} \\
\mathrm{C}\end{array}$ & $\begin{array}{l}\{1\} \\
\{2\} \\
\{3\} \\
\{4\} \\
\{5\} \\
\{6\} \\
\{7\} \\
\{8\} \\
\{9\}\end{array}$ \\
\hline$S S B$ & $\begin{array}{l}e e \rightarrow W^{+} W^{-} \\
e e \rightarrow W^{+} W^{-} \nu \bar{\nu} / Z^{0} Z^{0} \nu \bar{\nu}\end{array}$ & $\begin{array}{l}0.5 \\
1.0 \\
\end{array}$ & $\sigma$ & $\begin{array}{l}\Delta \kappa_{\gamma}, \lambda_{\gamma}=2 \cdot 10^{-4} \\
\Lambda_{* 4}, \Lambda_{* 5}=3 \mathrm{TeV}\end{array}$ & $\begin{array}{l}\mathrm{V} \\
\mathrm{C} \\
\end{array}$ & $\begin{array}{l}\{10\} \\
\{11\}\end{array}$ \\
\hline$S U S Y$ & $\begin{array}{l}e e \rightarrow \tilde{e}_{R}^{+} \tilde{e}_{R}^{-} \text {(Point 1) } \\
e e \rightarrow \tilde{\tau}_{1}^{+} \tilde{\tau}_{1}^{-}, \tilde{\chi}_{1}^{+} \tilde{\chi}_{1}^{-} \text {(Point 1) } \\
e e \rightarrow \tilde{t}_{1} \tilde{t}_{1} \text { (Point 1) }\end{array}$ & $\begin{array}{l}0.5 \\
0.5 \\
1.0\end{array}$ & $\begin{array}{l}E_{e} \\
E_{\pi}, E_{2 \pi}, E_{3 \pi}\end{array}$ & $\begin{array}{l}\delta M_{\tilde{\chi}_{1}^{0}}=50 \mathrm{MeV} \\
\delta\left(M_{\tilde{\tau}_{1}}-M_{\tilde{\chi}_{1}^{0}}\right)=200 \mathrm{MeV} \\
\delta M_{\tilde{t}_{1}}=2 \mathrm{GeV}\end{array}$ & $\begin{array}{l}\mathrm{T} \\
\mathrm{T}\end{array}$ & $\begin{array}{l}\{12\} \\
\{13\} \\
\{14\}\end{array}$ \\
\hline$-C D M$ & 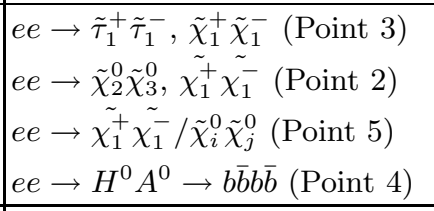 & \begin{tabular}{|l|}
0.5 \\
0.5 \\
$0.5 / 1.0$ \\
1.0 \\
\end{tabular} & $\begin{array}{l}M_{j j} \text { in } j j \not E, M_{\ell \ell} \text { in } j j \ell \ell \notin E \\
Z Z \not E, W W \notin \\
\text { Mass constrained } M_{b b} \\
\end{array}$ & $\begin{array}{l}\delta M_{\tilde{\tau}_{1}}=1 \mathrm{GeV}, \delta M_{\tilde{\chi}_{1}^{0}}=500 \mathrm{MeV} \\
\delta \sigma_{\tilde{\chi}_{2} \tilde{\chi}_{3}}=4 \%, \delta\left(M_{\tilde{\chi}_{2}^{0}}-M_{\tilde{\chi}_{1}^{0}}\right)=500 \mathrm{MeV} \\
\delta \sigma_{\tilde{\chi} \tilde{\chi}}=10 \%, \delta\left(M_{\tilde{\chi}_{3}^{0}}-M_{\tilde{\chi}_{1}^{0}}\right)=2 \mathrm{GeV} \\
\delta M_{A}=1 \mathrm{GeV}\end{array}$ & $\begin{array}{l}\text { F } \\
\text { C } \\
\text { C } \\
\text { C }\end{array}$ & $\begin{array}{l}\{15\} \\
\{16\} \\
\{17\} \\
\{18\}\end{array}$ \\
\hline $\begin{array}{l}\text { - alternative } \\
\text { SUSY } \\
\text { breaking }\end{array}$ & $\begin{array}{l}e e \rightarrow \tilde{\tau}_{1}^{+} \tilde{\tau}_{1}^{-}(\text {Point } 6) \\
\left.\tilde{\chi}_{1}^{0} \rightarrow \gamma+\not \mathcal{E} \text { (Point } 7\right) \\
\tilde{\chi}_{1}^{ \pm} \rightarrow \tilde{\chi}_{1}^{0}+\pi_{\text {soft }}^{ \pm}(\text {Point } 8)\end{array}$ & $\begin{array}{l}0.5 \\
0.5 \\
0.5\end{array}$ & $\begin{array}{l}\text { Heavy stable particle } \\
\text { Non-pointing } \gamma \\
\text { Soft } \pi^{ \pm} \text {above } \gamma \gamma \text { bkgd } \\
\end{array}$ & $\begin{array}{l}\delta M_{\tilde{\tau}_{1}} \\
\delta c \tau=10 \% \\
5 \sigma \text { Evidence for } \Delta \tilde{m}=0.2-2 \mathrm{GeV}\end{array}$ & $\begin{array}{l}\mathrm{T} \\
\mathrm{C} \\
\mathrm{F}\end{array}$ & $\begin{array}{l}\{19\} \\
\{20\} \\
\{21\}\end{array}$ \\
\hline $\begin{array}{l}\text { Precision SM } \\
\text { New Physics }\end{array}$ & $\begin{array}{l}e e \rightarrow t \bar{t} \rightarrow 6 \text { jets } \\
e e \rightarrow f \bar{f}(f=e, \mu, \tau ; b, c) \\
e e \rightarrow \gamma G(\mathrm{ADD}) \\
e e \rightarrow K K \rightarrow f \bar{f}(\mathrm{RS})\end{array}$ & $\begin{array}{l}1.0 \\
1.0 \\
1.0 \\
1.0\end{array}$ & $\begin{array}{l}\sigma_{f \bar{f}}, A_{F B}, A_{L R} \\
\sigma(\gamma+\not{f})\end{array}$ & $\begin{array}{l}5 \sigma \text { Sensitivity for }(g-2)_{t} / 2 \leq 10^{-3} \\
5 \sigma \text { Sensitivity to } M_{Z_{L R}}=7 \mathrm{TeV} \\
5 \sigma \text { Sensitivity }\end{array}$ & $\begin{array}{l}\mathrm{V} \\
\mathrm{V} \\
\mathrm{C} \\
\mathrm{T}\end{array}$ & $\begin{array}{l}\{22\} \\
\{23\} \\
\{24\} \\
\{25\}\end{array}$ \\
\hline $\begin{array}{l}\text { Energy/Lumi } \\
\text { Meas. }\end{array}$ & $\begin{array}{l}e e \rightarrow e e_{f w d} \\
e e \rightarrow Z^{0} \gamma\end{array}$ & $\begin{array}{l}0.3 / 1.0 \\
0.5 / 1.0\end{array}$ & & $\delta M_{\text {top }}=50 \mathrm{MeV}$ & $\begin{array}{l}\mathrm{T} \\
\mathrm{T}\end{array}$ & $\begin{array}{l}\{26\} \\
\{27\}\end{array}$ \\
\hline
\end{tabular}


can resolve combinatoric ambiguities in the reconstruction of $e^{+} e^{-} \rightarrow W^{+} W^{-}$and $e^{+} e^{-} \rightarrow t \bar{t}$. It will also thus extend the energy scale of the new physics that can be probed through anomalous coupling measurements as well as through the measurement of the forward-backward left-right asymmetries in $e^{+} e^{-} \rightarrow b \bar{b}, c \bar{c}$.

\section{B. Tracking Momentum Resolution}

The tracker momentum resolution is tested by the requirements of the recoil mass analysis of the Higgs-strahlung process $e^{+} e^{-} \rightarrow Z h \rightarrow \ell^{+} \ell^{-} X$. This analysis is used to measure the cross section for $e^{+} e^{-} \rightarrow Z h$ and the branching fraction of the dominant Higgs decay mode. It also provides the best Higgs mass measurement when there is a large branching ratio for inclusive Higgs decays to invisible particles. The possibility of measuring the suppressed $h^{0} \rightarrow \mu^{+} \mu^{-}$decay also sets an interesting benchmark on the tracking momentum resolution.

The measurement error for the slepton and lightest neutralino mass via the energy endpoint spectrum in $e^{+} e^{-} \rightarrow$ $\tilde{\ell}^{+} \tilde{\ell}^{-} \rightarrow \tilde{\chi}_{1}^{0} \tilde{\chi}_{1}^{0} \ell^{+} \ell^{-}$is also sensitive to the tracker momentum resolution when the decay lepton energy is greater than $100 \mathrm{GeV}$.

\section{Tracking Pattern Recognition}

Detection of long-lived neutral particles offers specific challenges to track pattern recognition. In $e^{+} e^{-} \rightarrow Z Z$, $W W, h Z$ and $H A$, between $50 \%$ and $65 \%$ of the events contain at least one $K_{S}^{0}$ or $\Lambda^{0}$. It is not uncommon for these particles to carry a significant amount of the jet energy. The jet energy resolution will thus be compromised if the charged tracks from $K_{S}^{0}$ or $\Lambda^{0}$ decay are missed or misinterpreted in the tracker pattern recognition.

Several key reactions have large jet multiplicities. More generally, quarks in the final state often lead to low momentum spiralling particles and to highly collimated jets with large local track densitites. These features test pattern recognition and track element linking in dense environments.

\section{Forward Tracking}

Multiple scattering significantly degrades the tracker momentum resolution in the forward region. Analyses of forward processes such as $e^{+} e^{-} \rightarrow e^{+} e^{-}, \mu^{+} \mu^{-}, Z \gamma, \nu_{e} \bar{\nu}_{e} h$ will therefore be particularly sensitive to the amount of material in the forward part of the tracker volume. The observation of the suppressed $h \rightarrow \mu \mu$ decay in $e^{+} e^{-} \rightarrow \nu_{e} \bar{\nu}_{e} h$ at $\sqrt{s}=1 \mathrm{TeV}$ is an excellent example of a measurement requiring good forward tracking momentum resolution, since the $M_{\mu \mu}$ signal must be kept as narrow as possible to be recognised above the $\nu_{e} \bar{\nu}_{e} Z^{*}$ background.

Beamline instrumentation will provide estimates of the energy, luminosity, and polarization of the electron and positron beams before and/or after collision, but the quantities of most relevance to physics analyses are the luminosity-weighted beam energy, luminosity and polarization. These luminosity-weighted quantities can only be obtained directly through measurements of forward processes such as $e^{+} e^{-} \rightarrow e^{+} e^{-}, Z \gamma$. Efficient forward tracking is especially important to reconstruct the luminosity spectrum from Bhabha scattering. Good forward momentum resolution is needed for both reactions.

Examples of physics processes requiring excellent beam energy and/or luminosity measurments include the threshold production of the top quark and the production of a Kaluza-Klein resonance $e^{+} e^{-} \rightarrow K K \rightarrow f \bar{f}$. As LEP was

able to do for the mass and width of the $Z$ boson at LEP, the ILC will be able to make a precision measurement of the mass and width of a KK resonance, but only if a very accurate beam energy measurement is available. 


\section{E. Low Momentum Leptons}

The region of SUSY parameter space in which the dark matter relic density is determined by slepton co-annihilation motivates models with a near-degeneracy of sleptons and neutralinos. These models give very soft final state leptons from $e^{+} e^{-} \rightarrow \tilde{\ell}^{+} \tilde{\ell}^{-} \rightarrow \tilde{\chi}_{1}^{0} \tilde{\chi}_{1}^{0} \ell^{+} \ell^{-}$. Such reactions challenge the tracker's ability to efficiently reconstruct charge tracks with very low $p_{T}$ and to identify very low momentum leptons. In some cases, the lepton momenta may be below the kinematic cut-off for the particle to reach the calorimeters or the muon detectors.

\section{F. Calorimeter}

At LEP2, hadronically decaying $W$ and $Z$ bosons were reconstructed using kinematic fits to improve the twojet mass resolution. Kinematic constraints were also used to improve the mass resolution of candidate $Z$ and $h$ bosons in searches for $e^{+} e^{-} \rightarrow Z h$. At the ILC, kinematic fits will continue to be useful for 4-fermion processes such as $e^{+} e^{-} \rightarrow W^{+} W^{-}, Z Z, Z h \rightarrow q \bar{q} q \bar{q}, l^{+} l^{-} q \bar{q}$, but they will have limited utility in 6 and 8 -fermion processes such as $e^{+} e^{-} \rightarrow Z h h, t \bar{t}, t \bar{t} h$, and will fail altogether for processes with final state neutrinos such as $e^{+} e^{-} \rightarrow$ $\nu_{e} \bar{\nu}_{e} h, \nu_{e} \bar{\nu}_{e} W^{+} W^{-}, \nu_{e} \bar{\nu}_{e} Z Z$.

The need to distinguish hadronically decaying $W, Z$, and $h$ bosons from one another without the benefit of kinematic constraints drives the present ILC jet energy resolution specification of $\Delta E_{j e t}=30 \% / \sqrt{E_{j e t}}$. Such a resolution cannot be obtained with the calorimeter alone. With the particle flow algorithm approach adopted by ILC detector groups, the jet energy is calculated by combining the sum of the tracker momenta of charged particles with the sum of the energies of showers not associated with charged particles. In this approach, a premium is placed on separating charged particle showers showers initiated by photons and by neutral hadrons. This leads to strong requirements on the calorimeter parameters such as the inner radius, the radiator material, the pixel size, and the number of layers.

\section{G. $\quad W, Z, h$ Boson Separation}

In the study of the strong symmetry breaking processes $e^{+} e^{-} \rightarrow \nu_{e} \bar{\nu}_{e} W^{+} W^{-}, \nu_{e} \bar{\nu}_{e} Z Z$ and in SUSY processes such as $e^{+} e^{-} \rightarrow \tilde{\chi}_{1}^{+} \tilde{\chi}_{1}^{-} \rightarrow \tilde{\chi}_{1}^{0} \tilde{\chi}_{1}^{0} W^{+} W^{-}$and $e^{+} e^{-} \rightarrow \tilde{\chi}_{2}^{0} \tilde{\chi}_{2}^{0} \rightarrow \tilde{\chi}_{1}^{0} \tilde{\chi}_{1}^{0} Z Z$, it is important to distinguish the $W^{+} W^{-}$ final state from $Z Z$. In the $W W$ fusion production of Higgs, $e^{+} e^{-} \rightarrow \nu_{e} \bar{\nu}_{e} h$, the Higgs signal must be separated from a background of $e^{+} e^{-} \rightarrow e^{-} \bar{\nu}_{e} W^{+}, e^{+} e^{-} Z, \nu_{e} \bar{\nu}_{e} Z$ by the direct reconstruction of the Higgs mass. The signal for invisible decays of the Higgs, $e^{+} e^{-} \rightarrow Z h \rightarrow q \bar{q}+\not$, is obtained by requiring that the reconstructed $q \bar{q}$ mass be close to the $Z$ mass. and that the recoil mass obtained from the $q \bar{q} 2$-jet system be close to the Higgs mass. When measuring the Higgs boson self-coupling, the signal for the $W W$ fusion production of two Higgs bosons, $e^{+} e^{-} \rightarrow \nu_{e} \bar{\nu}_{e} h h$, must be separated from a background of $e^{+} e^{-} \rightarrow \nu_{e} \bar{\nu}_{e} W^{+} W^{-}, \nu_{e} \bar{\nu}_{e} Z Z, e^{-} \bar{\nu}_{e} Z W^{+}$, etc. In all of these cases the jet energy resolution plays a central role.

\section{H. Multi-Jet Combinatorics}

Good calorimetry can help reduce the combinatorics in reactions with four or more jets. Examples for which this is particularly important are the measurements of the Higgs branching fraction to $W W^{*}$ using $e^{+} e^{-} \rightarrow Z h \rightarrow$ $Z W W^{*} \rightarrow q \bar{q} q \bar{q} l \nu$, the measurement of the Higgs self-coupling using $e^{+} e^{-} \rightarrow Z h h \rightarrow q \bar{q} b \bar{b} b \bar{b}$, and the measurement of the top quark Yukawa coupling using $e^{+} e^{-} \rightarrow t \bar{t} h \rightarrow b \bar{b} b \bar{b} q \bar{q} q \bar{q}$. In all of these cases, the likelihood that jets will be correctly assigned to their parent particles will depend on how well the calorimeter reconstructs $W Z$, and $h$ boson masses. 
TABLE III: Table of relations between the benchmark physics processes and parameters of detector subsystems

\begin{tabular}{|c|c|c|c|c|c|c|c|c|c|c|c|c|c|c|}
\hline \multirow[t]{2}{*}{ Process } & \multirow{2}{*}{\begin{tabular}{|c|} 
Vertex \\
$\sigma_{I P}$ \\
\end{tabular}} & \multicolumn{2}{|c|}{ Tracking } & \multicolumn{2}{|c|}{ Calorimetry } & \multicolumn{2}{|c|}{ Fwd } & \multirow{2}{*}{$\frac{\text { Very Fwd }}{\theta_{\min }^{e}}$} & \multicolumn{5}{|c|}{ Integration } & \multirow[t]{2}{*}{ Pol. } \\
\hline & & $\delta p / p^{2}$ & $\bar{\epsilon}$ & $\delta E$ & $\delta \theta, \delta \phi$ & Trk & $\mathrm{Cal}$ & & $\delta E_{\text {jet }}$ & $M_{j j}$ & $\ell$-Id & $V^{0}$-Id & $Q_{\text {jet } / v t x}$ & \\
\hline$e e \rightarrow Z h \rightarrow \ell \ell X$ & & $\mathrm{x}$ & & & & & & & & & $\mathrm{x}$ & & & \\
\hline$e e \rightarrow Z h \rightarrow j j b b$ & $\mathrm{x}$ & $\mathrm{x}$ & $\mathrm{x}$ & & & $\mathrm{x}$ & & & & $\mathrm{x}$ & $\mathrm{x}$ & & & \\
\hline$e e \rightarrow Z h, h \rightarrow b b / c c / \tau \tau$ & $\mathrm{x}$ & & $\mathrm{x}$ & & & & & & & $\mathrm{x}$ & $\mathrm{x}$ & & & \\
\hline$e e \rightarrow Z h, h \rightarrow W W$ & $\mathrm{x}$ & & $\mathrm{x}$ & & $\mathrm{x}$ & & & & $\mathrm{x}$ & $\mathrm{x}$ & $\mathrm{x}$ & & & \\
\hline$e e \rightarrow Z h, h \rightarrow \mu \mu$ & $\mathrm{x}$ & $\mathrm{x}$ & & & & & & & & & $\mathrm{x}$ & & & \\
\hline$e e \rightarrow Z h, h \rightarrow \gamma \gamma$ & & & & $\mathrm{x}$ & $\mathrm{x}$ & & $\mathrm{x}$ & & & & & & & \\
\hline$e e \rightarrow Z h, h \rightarrow$ invisible & & & $\mathrm{x}$ & & & $\mathrm{x}$ & $\mathrm{x}$ & & & & & & & \\
\hline$e e \rightarrow \nu \nu h$ & $\mathrm{x}$ & $\mathrm{x}$ & $\mathrm{x}$ & $\mathrm{x}$ & & & $\mathrm{x}$ & & & $\mathrm{x}$ & $\mathrm{x}$ & & & \\
\hline$e e \rightarrow t t h$ & $\mathrm{x}$ & $\mathrm{x}$ & $\mathrm{x}$ & $\mathrm{x}$ & $\mathrm{x}$ & & $\mathrm{x}$ & $\mathrm{x}$ & $\mathrm{x}$ & & $\mathrm{x}$ & & & \\
\hline$e e \rightarrow Z h h, \nu \nu h h$ & $\mathrm{x}$ & $\mathrm{x}$ & $\mathrm{x}$ & $\mathrm{x}$ & $\mathrm{x}$ & $\mathrm{x}$ & $\mathrm{x}$ & & $\mathrm{x}$ & $\mathrm{x}$ & $\mathrm{x}$ & $\mathrm{x}$ & $\mathrm{x}$ & $\mathrm{x}$ \\
\hline $\begin{array}{l}e e \rightarrow W W \\
e e \rightarrow \nu \nu W W / Z Z\end{array}$ & & & & & & $\mathrm{x}$ & $\mathrm{x}$ & & $\mathrm{x}$ & $\begin{array}{l}\mathrm{x} \\
\mathrm{x}\end{array}$ & $\mathrm{x}$ & & $\mathrm{x}$ & \\
\hline$e e \rightarrow \tilde{e}_{R} \tilde{e}_{R}$ (Point 1) & & $\mathrm{x}$ & & & & & & $\mathrm{x}$ & & & $\mathrm{x}$ & & & $\mathrm{x}$ \\
\hline$e e \rightarrow \tilde{\tau}_{1} \tilde{\tau}_{1}$ & $\mathrm{x}$ & $\mathrm{x}$ & & & & & & $\mathrm{x}$ & & & & & & \\
\hline$e e \rightarrow \tilde{t}_{1} \tilde{t}_{1}$ & $\mathrm{x}$ & $\mathrm{x}$ & & & & & & & $\mathrm{x}$ & $\mathrm{x}$ & & $\mathrm{x}$ & & \\
\hline$e e \rightarrow \tilde{\tau}_{1} \tilde{\tau}_{1}$ (Point 3$)$ & $\mathrm{x}$ & $\mathrm{x}$ & & & $\mathrm{x}$ & $\mathrm{x}$ & $\mathrm{x}$ & $\mathrm{x}$ & $\mathrm{x}$ & & & & & \\
\hline$e e \rightarrow \tilde{\chi}_{2}^{0} \tilde{\chi}_{3}^{0}$ (Point 5) & & & & & & & & & $\mathrm{x}$ & $\mathrm{x}$ & & & & \\
\hline$e e \rightarrow H A \rightarrow b b b b$ & $\mathrm{x}$ & $\mathrm{x}$ & & & & & & & & $\mathrm{x}$ & $\mathrm{x}$ & & & \\
\hline$e e \rightarrow \tilde{\tau}_{1} \tilde{\tau}_{1}$ & & & $\mathrm{x}$ & & & & & & & & & & & \\
\hline$\tilde{\chi}_{1}^{0} \rightarrow \gamma+\not E$ & & & & & $\mathrm{x}$ & & & & & & & & & \\
\hline$\tilde{\chi}_{1}^{ \pm} \rightarrow \tilde{\chi}_{1}^{0}+\pi_{\text {soft }}^{ \pm}$ & & & $\mathrm{x}$ & & & & & $\mathrm{x}$ & & & & & & \\
\hline$e e \rightarrow t t \rightarrow 6$ jets & $\mathrm{x}$ & & $\mathrm{x}$ & & & & & & $\mathrm{x}$ & $\mathrm{x}$ & $\mathrm{x}$ & & & \\
\hline$e e \rightarrow f f[e, \mu, \tau ; b, c]$ & $\mathrm{x}$ & & $\mathrm{x}$ & & & & $\mathrm{x}$ & & $\mathrm{x}$ & & $\mathrm{x}$ & & $\mathrm{x}$ & $\mathrm{x}$ \\
\hline$e e \rightarrow \gamma G(\mathrm{ADD})$ & & & & $\mathrm{x}$ & $\mathrm{x}$ & & & $\mathrm{x}$ & & & & & & $\mathrm{x}$ \\
\hline$e e \rightarrow K K \rightarrow f \bar{f}$ & & $\mathrm{x}$ & & & & & & & & & $\mathrm{x}$ & & & \\
\hline$e e \rightarrow e e_{f w d}$ & & & & & & $\mathrm{x}$ & $\mathrm{x}$ & $\mathrm{x}$ & & & & & & \\
\hline$e e \rightarrow Z \gamma$ & & $\mathrm{x}$ & & $\mathrm{x}$ & $\mathrm{x}$ & $\mathrm{x}$ & $\mathrm{x}$ & & & & & & & \\
\hline
\end{tabular}

\section{Heavy Particle Mass Measurements}

Although kinematic constraints can be used to significantly improve the Higgs mass resolution in the processes $e^{+} e^{-} \rightarrow Z h \rightarrow q \bar{q} b \bar{b}$ and $e^{+} e^{-} \rightarrow H A \rightarrow b \bar{b} b \bar{b}$, the calorimeter performance may still play an important role in determining the ultimate Higgs mass precision.

\section{J. Photons}

In the strategy of particle flow calorimeter, the electromagnetic calorimeter should be designed to optimize the separation of charged showers from neutral showers. However, it is important in doing this not to overlook traditional calorimeter figures of merit such as the intrinsic resolution for electromagetic showers. An example of a measurement requiring very good electromagnetic calorimeter energy resolution is the measurement of the Higgs branching ratio to two photons. Initial measurements of this branching ratio can be performed at $\sqrt{s}=350 \mathrm{GeV}$ using the Higgsstrahlung process; later the error on the branching fraction can be improved using $e^{+} e^{-} \rightarrow \nu_{e} \bar{\nu}_{e} h$ at $\sqrt{s}=1 \mathrm{TeV}$.

The ability to efficiently detect soft photons over most of the solid angle is important for the measurement of 
graviton production in association with a photon, $e^{+} e^{-} \rightarrow \gamma G$, seen as $\gamma+E$

The fine granularity of an ILC calorimeter can help identify photons that do not point back to the interaction point. For example, in GMSB models of SUSY the neutralino may decay inside the detector via $\tilde{\chi}_{1}^{0} \rightarrow \gamma+\not$, producing two photons appearing in the detector superimposed on each SUSY event.

\section{K. Far Forward Detector}

The far forward detector should be able to veto electrons down to an angle of a few mrads in the presence of a large $e^{+} e^{-}$pair background. This capability is needed in general to suppress background from $\gamma \gamma \rightarrow f \bar{f}$ in any analysis with missing energy in the final state. It takes on added importance given the possiblity that some SUSY particles may be nearly degenerate with the LSP. Examples of processes requiring superlative rejection of the $\gamma \gamma \rightarrow f \bar{f}$ background include $e^{+} e^{-} \rightarrow \tilde{\tau}_{1} \tilde{\tau}_{1} \rightarrow \tilde{\chi}_{1}^{0} \tilde{\chi}_{1}^{0} \tau^{+} \tau^{-}$and $e^{+} e^{-} \rightarrow \tilde{\chi}_{1}^{+} \tilde{\chi}_{1}^{-} \rightarrow \tilde{\chi}_{1}^{0} \tilde{\chi}_{1}^{0} \pi^{+} \pi^{-}$where the $\tilde{\tau}_{1}$ or $\tilde{\chi}_{1}^{+}$is nearly degenerate with the $\tilde{\chi}_{1}^{0}$.

\section{SELECTING A REDUCED BENCHMARK LIST}

The broad set of physics reactions identified above provides detailed coverage of the detector performance requirements for an ILC detector. However, the list of reactions is rather long. Thus, it is useful to call out a smaller subset of benchmarks which emphasize key aspects of the detector performances - vertexing, tracking, calorimetry, very forward instrumentation and integration. There are certainly several such subsets which could be chosen. In our choice, we give special weight to those reactions whose analysis maintains a simple relation between the physics measurement and the basic detector parameters. We also give weight to those reactions that are key to the ILC physics case and emphasise the extended capabilities that the ILC will provide with respect to the LHC.

The Higgs sector offers several reactions. The process $e^{+} e^{-} \rightarrow Z h \rightarrow \ell \ell X$ tests momentum resolution for energetic, isolated charged particle tracks. It presents a challenging accuracy target for $\sigma_{h Z}$, which is justified because this represents the common normalisation for the extraction of Higgs couplings. The Higgs branching fraction measurements $e^{+} e^{-} \rightarrow Z h, h \rightarrow c c, \tau \tau, W W^{*}$ probe the detector capability in tagging heavy flavours, which drives the vertex performance. In particular, it gives stringent tests of charm di-jet tagging under a dominant $b \bar{b}$ background, and of $\tau$ tagging by single particle impact parameter measurement. The reaction $e^{+} e^{-} \rightarrow Z h h$, which give access to the Higgs self-coupling and the reconstruction of the Higgs potential, tests energy flow and di-jet mass resolution to discriminate the $Z h h$ signal from the $Z Z Z$ background. It also tests $b$ tagging efficiency in complex six-jet final states, and it provides an example in which vertex charge can be used to resolve combinatoric ambiguities in jet assignment.

Among the variety of SUSY reactions, $e^{+} e^{-} \rightarrow \tilde{e}_{R} \tilde{e}_{R}$ gives a sharp test of lepton tagging and momentum resolution. The reaction $e^{+} e^{-} \rightarrow \tilde{\tau}_{1} \tilde{\tau}_{1}$ in the co-annihilation region of cosmologically interesting supersymmetric parameters offers severe challenges to charged particle detection, due to the small splitting between the $\tilde{\tau}_{1}$ and the $\tilde{\chi}_{1}^{0}$, and to the design of the far forward region, due to the need to detect forward electrons to suppress the $e^{+} e^{-} \rightarrow e^{+} e^{-} \tau^{+} \tau^{-}$two-photon background. Reactions containing the decays $\tilde{\chi}_{1}^{ \pm} \rightarrow W^{ \pm} \tilde{\chi}_{1}^{0}$ and $\tilde{\chi}_{2}^{0} \rightarrow Z^{0} \tilde{\chi}_{1}^{0}$ test the energy flow performance through on-shell $W$ and $Z$ identification and reconstruction.

Two-fermion final states at the highest energy provide an excellent test of tracking and calorimetric performances with high local particle density. The study of $e^{+} e^{-} \rightarrow f \bar{f}$ may offer a valuable window on physics well beyond the $\sqrt{s}$ limit by electroweak fits to precision observables. This study requires good $b$ and $c$ jet flavor tagging, in an almost democratic flavor mixture, and measurements of jet and vertex charge for computing forward-backward asymmetries. Polarization-dependence is large, so the analysis also benefits from the use and precision measurement of beam polarization.

Finally, samples of single particles, produced over the whole polar angle and a wide energy range, and di-jet events will complement these benchmarks and highlight the deterioration in efficiency and resolution for particles 
in jets and complex events. These samples will enable tests of reconstruction in a simple environment. They will provide a basis for defining the detector response in terms of efficiency and resolution and will produce well-motivated parametrizations to be used in physics studies carried out with fast simulation.

In this way, we have arrived at the following proposal for a reduced list of benchmark reactions for initial study. Our list includes reactions that test all elements from the menu of basic detector capabilities.

0. Single $e^{ \pm}, \mu^{ \pm}, \pi^{ \pm}, \pi^{0}, K^{ \pm}, K_{S}^{0}, \gamma, 0<|\cos \theta|<1,0<p<500 \mathrm{GeV}$

1. $e^{+} e^{-} \rightarrow f \bar{f}, f=e, \tau, u, s, c, b$ at $\sqrt{s}=0.091,0.35,0.5$ and $1.0 \mathrm{TeV}$;

2. $e^{+} e^{-} \rightarrow Z^{0} h^{0} \rightarrow \ell^{+} \ell^{-} X, M_{h}=120 \mathrm{GeV}$ at $\sqrt{s}=0.35 \mathrm{TeV}$;

3. $e^{+} e^{-} \rightarrow Z^{0} h^{0}, h^{0} \rightarrow c \bar{c}, \tau^{+} \tau^{-}, W W^{*}, M_{h}=120 \mathrm{GeV}$ at $\sqrt{s}=0.35 \mathrm{TeV}$;

4. $e^{+} e^{-} \rightarrow Z^{0} h^{0} h^{0}, M_{h}=120 \mathrm{GeV}$ at $\sqrt{s}=0.5 \mathrm{TeV}$;

5. $e^{+} e^{-} \rightarrow \tilde{e}_{R}^{+} \tilde{e}_{R}^{-}$at Point 1 at $\sqrt{s}=0.5 \mathrm{TeV}$;

6. $e^{+} e^{-} \rightarrow \tilde{\tau}_{1}^{+} \tilde{\tau}_{1}^{-}$, at Point 3 at $\sqrt{s}=0.5 \mathrm{TeV}$;

7. $e^{+} e^{-} \rightarrow \tilde{\chi}_{1}^{+} \tilde{\chi}_{1}^{-} / \tilde{\chi}_{2}^{0} \tilde{\chi}_{2}^{0}$ at Point 5 at $\sqrt{s}=0.5 \mathrm{TeV}$;

This list of reactions seems still to be rather lengthy, especially since each item contains a number of distinct physics measurements. However, we believe that, for the initial stages of detector design and optimization, this set of physics topics can be surveyed at a useful level. In preparing this list, we have been careful to include only reactions that have already been studied extensively and for which established analysis algorithms exist. These algorithms, based on reconstructed physics objects such as electrons, muons, charged hadrons, photons, and neutral hadrons, are to a very good approximation independent of the details of the detector design. In fact, they could be standardized and shared among the detector concept groups. Once a concept group has developed the software needed to convert fully simulated raw data to reconstructed electrons, muons, charged hadrons, photons, and neutral hadrons, it can utilize the common standardized physics algorithms to quickly survey the physics capability of its design. Analyses at this level, while not achieving the ultimate optimized performance for each detector, would come close enough to guide the detector design process and also to realistically predict the physics capabilities of the ILC experiments.

\section{CONCLUSIONS}

In this note, we have reviewed benchmark physics processes for the ILC that give broad coverage of the requirements that ILC detectors must satisfy. We have described the mapping between these reactions and the capabilties they require in the various detector subsystems. Finally, we have provided guidelines for the selection of smaller sets of benchmarks for the early stages of detector development, and we have suggested an especially economical subset on which to begin the detector performance studies.

\section{Acknowledgments}

This paper has been prepared on request of the ILC Worldwide Study. The authors wish to thank Hitoshi Yamamoto for extensive discussion and Yasuhiro Sugimoto and Graham Wilson for their contributions to the LCWS05 benchmark parallel session. The work of M.B. was supported by the Director, Office of Science, Office of Basic Energy Sciences, of the U.S. Department of Energy under Contract No. DE-AC02-05CH11231. The work of T.B. and M.P. has been supported by the US Department of Energy, contract DE-AC02-76SF00515. The work of Y.O. has been 
supported by a Grant-in-Aid of the Ministry of Education, Culture, Sports, Science, and Technology, Government of Japan, Nos. 13640309, 13135225, 16081211, and 17540286.

[1] See http://www.ilcldc.org/

[2] See http://www-sid.slac.stanford.edu/

[3] See http://ilcphys.kek.jp/gld/

[4] F. E. Paige, S. D. Protopescu, H. Baer and X. Tata, arXiv hep-ph/0312045

[5] B. C. Allanach et al., in Proc. of the APS/DPF/DPB Summer Study on the Future of Particle Physics (Snowmass 2001) ed. N. Graf, Eur. Phys. J. C 25 (2002) 113 [eConf C010630, P125 (2001)] [arXiv hep-ph/0202233] and update in J. A. Aguilar-Saavedra et al., arXiv hep-ph/0511344

[6] M. Battaglia, J. Feng, N. Graf, M Peskin, M. Trodden (editors), White paper on ILC - Cosmology connections, in preparation.

[7] M. Battaglia, A. De Roeck, J. R. Ellis, F. Gianotti, K. A. Olive and L. Pape, Eur. Phys. J. C 33, 273 (2004) [arXiv hep-ph/0306219.

[8] A. De Roeck, J. R. Ellis, F. Gianotti, F. Moortgat, K. A. Olive and L. Pape, arXiv hep-ph/0508198

[9] B. Aubert et al. [BABAR Collaboration], Phys. Rev. Lett. 93, 011803 (2004) [arXiv hep-ex/0404017.

[10] O. Buchmuller and H. Flacher, arXiv hep-ph/0507253

[11] J. H. Kuhn and M. Steinhauser, Nucl. Phys. B 619, 588 (2001) [Erratum-ibid. B 640, 415 (2002)] [arXiv hep-ph/0109084].

[12] M. Battaglia and K. Desch, arXiv hep-ph/0101165

[13] E. Boos, J. C. Brient, D. W. Reid, H. J. Schreiber and R. Shanidze, Eur. Phys. J. C 19 (2001) 455 [arXiv hep-ph/0011366].

[14] T. L. Barklow, arXiv hep-ph/0312268

[15] M. Schumacher, LC-PHSM-2003-096

[16] M. Battaglia, E. Boos and W. M. Yao, in Proc. of the APS/DPF/DPB Summer Study on the Future of Particle Physics (Snowmass 2001) ed. N. Graf, eConf C010630 (2001) E3016 [arXiv hep-ph/0111276.

[17] Y. Yasui, S. Kanemura, S. Kiyoura, K. Odagiri, Y. Okada, E. Senaha and S. Yamashita, arXiv hep-ph/0211047

[18] S. Yamashita, to appear in the proceedings of the LCWS04 workshop.

[19] M. Kuroda et al., Phys. Lett. B138 (1987) 366.

[20] E. Boos, H. He, W. Kilian, A.Pukhov, C.P. Yuan and P.M. Zerwas, Phys. Rev. D57 (1988) 1553 [arXiv hep-ph/9708310.

[21] P. Krstonovic et al., to appear in the Proceedings of the LCWS05 Workshop, Stanford, March 2005.

[22] H. U. Martyn, arXiv hep-ph/0408226

[23] P. Bambade, M. Berggren, F. Richard and Z. Zhang, arXiv hep-ph/0406010

[24] R. Gray et al., arXiv hep-ex/0507008

[25] M. Battaglia, arXiv hep-ph/0410123

[26] J. L. Feng and B. T. Smith, Phys. Rev. D 71, 015004 (2005) [Erratum-ibid. D 71, 0109904 (2005)] [arXiv hep-ph/0409278.

[27] S. Ambrosiano and G.A. Blair, Eur. Phys. J. C12 287 (2000).

[28] J. F. Gunion and S. Mrenna, Phys. Rev. D 64, 075002 (2001) [arXiv hep-ph/0103167.

[29] S. Riemann, LC-TH-2001-007.

[30] G. F. Giudice, R. Rattazzi and J. D. Wells, Nucl. Phys. B 544 (1999) 3 [arXiv hep-ph/9811291.

[31] E. A. Mirabelli, M. Perelstein and M. E. Peskin, Phys. Rev. Lett. 82 (1999) 2236 [arXiv hep-ph/9811337.

[32] G. W. Wilson, LC-PHSM-2001-010

[33] H. Davoudiasl, J. L. Hewett and T. G. Rizzo, Phys. Rev. Lett. 84 (2000) 2080 [arXiv hep-ph/9909255].

[34] T. G. Rizzo, arXiv hep-ph/0409309

[35] S. T. Boogert and D. J. Miller, arXiv hep-ex/0211021 and refs. therein. 


\section{Notes to Table II}

$\{1\}$ The target is to determine the Higgs-strahlung cross section with $2.5 \%$ accuracy, in addition the Higgs mass should be obtained to $\delta \mathrm{M}_{h}=100 \mathrm{MeV}$.

$\{2\}$ The Higgs branching fractions can be extracted by a fit to the jet flavour tagging response on candidate $h^{0} Z^{0}$ events. The target accuracies correspond to the estimated theoretical accuracies assuming the $b$ and $c$ quark masses to be determined at $B$ factories to $\pm 50 \mathrm{MeV}$ and $\pm 30 \mathrm{MeV}$, respectively [9, 10] or alternatively from heavy quark production in low-energy $e^{+} e^{-}$ annihilation [11].

$\{3\}$ The target precision on $\operatorname{BR}\left(h^{0} \rightarrow W^{+} W^{-}\right)$is set by the requirement of comparable accuracies in $\Gamma_{h \rightarrow W}$ (measured in $W W$ fusion) and in the branching fraction, for the extraction of the Higgs total decay width 12 ].

\{4\} The analysis should be performed at $\sqrt{s}=1 \mathrm{TeV}$, in the sum of the $\gamma \gamma j j$ and $\gamma \gamma \nu \nu$, sensitive to both Higgs-strahlung and fusion production processes, as in [13, 14]. The target precision is set by requiring the Higgs total width to be determined, using the combination of $h \rightarrow \gamma \gamma$ and $\gamma \gamma \rightarrow h$ at a photon collider, to an accuracy comparable to that using the $W W$ coupling (see note $\{3\})$.

$\{5\}$ Observe a $>5 \sigma$ signal of $h \rightarrow \mu \mu$ assuming SM couplings for a $M_{h}=120 \mathrm{GeV}$.

$\{6\}$ The target is to obtain a $5 \sigma$ signal for $h \rightarrow$ invisible for an invisible branching fraction of $2.5 \%$, from a $j j \not E$ analysis 15$]$. \{7\} A challenge for $b$-tagging and $M_{j j}$ in a case where global kinematic fitting is not possible.

$\{8\}$ The target is to measure the top Yukawa coupling $g_{t t h}$ to a statistical accuracy of $5 \%$ from the analysis of $e^{+} e^{-} \rightarrow t \bar{t} h$ at $\sqrt{s}=1 \mathrm{TeV}$.

$\{9\}$ Improved techniques in double Higgs-strahlung and WW fusion and availability of polarised beams are expected to reduce the individual errors for the triple Higgs coupling to about $15 \%$ in the two channels so that the combined accuracy should finally reach the $10 \%$ limit $[16,17,18]$.

$\{10\}$ The analysis is carried out for exact $\mathrm{SU}(2) \times \mathrm{U}(1)$ invariance. The five CP-invariant static electroweak parameters of the $W^{ \pm}$bosons, i.e. the Z-charge, the two magnetic dipole and electric quadrupole moments can be expressed in terms of just three parameters $\Delta g_{Z}, \Delta \kappa_{Z}$ and $\lambda_{Z}$ as in Ref. [19].

$\{11\}$ Interactions among $W$ and $Z$ bosons in scenarios of strong electroweak symmetry breaking are generally described by chiral expansions. The expansion parameters, $\left\{v / \Lambda_{*}\right\}^{2}$, are expressed by strong interaction scales which may extend up to values of $4 \pi v=3 \mathrm{TeV}$. Beyond $W W$ energies of $3 \mathrm{TeV}$ resonances should be formed. In theories respecting $\mathrm{SU}(2)$ isospin invariance two parameters determine the amplitudes for [quasi-]elastic $W W \rightarrow W W, Z Z$ scattering, $\Lambda_{* 4}$ and $\Lambda_{* 5}$, see Ref. [20]. A $1 \mathrm{TeV}$ ILC ought to cover the entire $\Lambda_{* 4}$ and $\Lambda_{* 5}$ parameter region below $3 \mathrm{TeV}$. The reach refers to an analysis based on optimal observables [21].

\{12\} SUSY parameters of Point 1 of Table 1, corresponding to update of mSUGRA SPS1a/LCC 1 point [5, 6], now SPS1a'. The target is to extract the lightest neutralino mass to the specified accuracy from the analysis of the lepton energy spectrum, assuming to know the selectron mass to $\pm 50 \mathrm{MeV}$ from a dedicated threshold scan.

$\{13\}$ SUSY parameters of Point 1 of Table 1 , the $\tilde{\tau}_{1}$ to $\tilde{\chi}_{1}^{0}$ mass difference can be obtained by a study of the energy distribution of the hadronic system in the decays $\tilde{\tau}_{1}^{ \pm} \rightarrow \tilde{\chi}_{1}^{0} \tau^{ \pm}, \tau^{ \pm} \rightarrow \pi^{ \pm} \nu, \rho^{0} \nu, a_{1}^{ \pm} \nu$, which give one, two and three pion final states [22].

$\{14\}$ A challenge for precision kinematic endpoint measurement from jets.

\{15\} SUSY parameters in co-annihilation region, Point 3 of Table 1, corresponding to mSUGRA Point D' of [7]. The target accuracies on the $\tilde{\tau}$ and neutralino mass are required to determine $\Omega_{\chi} h^{2}$ to a statistical accuracy comparable to that of CMB experiments. The $\tilde{\tau}$ mass can be obtained to a $\pm 500 \mathrm{MeV}$ accuracy from a dedicated threshold scan. The $\tilde{\tau} \tilde{\tau} \rightarrow \tau \tilde{\chi} \tau \tilde{\chi}$ is contaminated by $e e \rightarrow \tau \tau e e$ which requires low angle $e$ tagging and, possibly, $\mu / \pi$ id in the very forward instrumentation 23 ]. $\{16\}$ SUSY parameters in focus point region, Point 2 of Table 1, corresponding to mSUGRA Point LCC 2 of [6]. Gauginos decays into ligther states and virtual $W$ and $Z$ bosons. The target accuracies on the gaugino mass differences are required to determine $\Omega_{\chi} h^{2}$ to a 5-10\% statistical accuracy. The mass differences can be extracted by the kinematical upper limit of the $M_{j j}$ and $M_{\ell \ell}$ in the $j j \nu \nu \tilde{\chi} \tilde{\chi}$ and $j j \ell \ell \tilde{\chi} \tilde{\chi}$ final states, respectively. The difference in the kinematics allows to distinguish

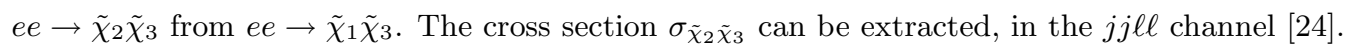

$\{17\}$ SUSY parameters for non universal Higgs masses (NHUM) model, from [8], Point 5 of Table 1 . This point has real $W$ and $Z$ bosons produced in heavier gaugino cascade decays.

\{18\} SUSY parameters in $A$ annihilation funnel, Point 4 of Table 1, correspondinding to mSUGRA Point LCC 4 of [6]. The target accuracy on $M_{A}$ is required to extract $\Omega_{\chi} h^{2}$ to $5-10 \%$ statistical accuracy [25].

\{19\} SUSY parameters for Point 6 of Table 1, from [8], with gravitino LSP and quasi-stable next-to-lightest supersymmetric particle [26]. Experimental signature is long-lived particle traversing the detector as heavy ionizing particle. The track curvature in the magnetic field can be used to determine the $\tilde{\tau}$ mass. The reference mass is $M_{\tilde{\tau}_{1}}=150 \mathrm{GeV}$. 
$\{20\}$ SUSY GMSB scenario, the final state particle in SUSY decay chain is the gravitino, albeit with a mass of less than $\mathrm{keV}$ [27]. The benchmark point parameters are $M_{\text {messenger }}=240 \mathrm{TeV}, \Lambda=110 \mathrm{TeV}, \sqrt{F}=0.8 \mathrm{PeV}, M_{\tilde{G}}=0.15 \mathrm{keV}, M_{\tilde{\chi}_{1}^{0}}$ $=150 \mathrm{GeV}, M_{\tilde{\tau}}=200 \mathrm{GeV}$.

$\{21\}$ SUSY AMSB scenario: in this scheme, the charginos $\tilde{\chi}_{1}^{ \pm}$are nearly mass degerate with the lightest neutralino $\tilde{\chi}_{1}^{0}$. Chargino pair production therefore generates two soft hadrons, viz. $e^{+} e^{-} \rightarrow \tilde{\chi}_{1}^{+} \tilde{\chi}_{1}^{-} \rightarrow \pi^{+} \pi^{-}+\not$, see Ref. [28]. SUSY parameters of point SPS9 [5]: $m_{0}=450 \mathrm{GeV}, m_{3 / 2}=60000 \mathrm{GeV}, \tan \beta=10, \operatorname{Sign}(\mu)=+1$ with $M_{\text {top }}=178 \mathrm{GeV}$, corresponding to $M_{\tilde{\chi}_{1}^{0}}=$ $180.88 \mathrm{GeV}$ and $M_{\tilde{\chi}_{1}^{ \pm}}=181.05 \mathrm{GeV}$ in ISAJET.7.69.

$\{23\}$ The accuracy in fermion pair production properties at ILC can be quantified by studying the mass reach for the heavy neutral vector boson $M_{Z_{L R}}$ in left-right symmetric extensions of the Standard Model. The virtual effects should be determined at ILC so well that the mass range should be tripled compared with direct production at LHC [29]. The $A_{F B}$ measurement is particularly sensitive to jet/vertex charge determination.

$\{24\}$ Single photon signature of $e e \rightarrow \gamma G$ in ADD scenario for number of extra dimensions $\delta=4, M_{D}=4 \mathrm{TeV}$, see Refs. 30 , 31] Beam polarization $\left(\mathcal{P}\left(e^{-}\right) \simeq 0.8, \mathcal{P}\left(e^{+}\right) \simeq 0.6\right)$ is needed for suppressing the $e e \rightarrow \nu \nu \gamma$ background [32].

$\{25\}$ Two fermion final states of Kaluza Klein graviton excitation production in Randall Sundrum model [33, 34]. The model parameters are $m_{1}=1 \mathrm{TeV}$ and $k / \bar{M}_{P l}=0.05$, where $k$ is the curvature parameter and $\bar{M}_{P l}$ the reduced Planck mass. The decay is implemented in Pythia, since version 6.2, at process ISUB $=391$. In version 6.3, this benchmark corresponds to $\operatorname{PMAS}(\operatorname{PYCOMP}(5000039), 1)=900 . \mathrm{d} 0$ and $\operatorname{PARP}(50)=0.05 \mathrm{~d} 0$. The aim is to determine the resonance mass and width and to test its decay branching fractions to leptons and quarks with accuracies comparable to those obtained at LEP/SLC for the $Z^{0}$.

$\{26\}$ Forward Bhabha scattering, partly acoplanar due to initial and final-state photon radiation, serves to measure energy and luminosity, accounting thereby for the effects of soft real photon radiation due to beamstrahlung [35]. Observed in situ, these measurements are a necessary complement to using external spectrometers for integral energy measurements before and after the bunch collisions.

$\{27\}$ Beam energy determination by the $e^{+} e^{-}$annihilation to $Z \gamma$. 